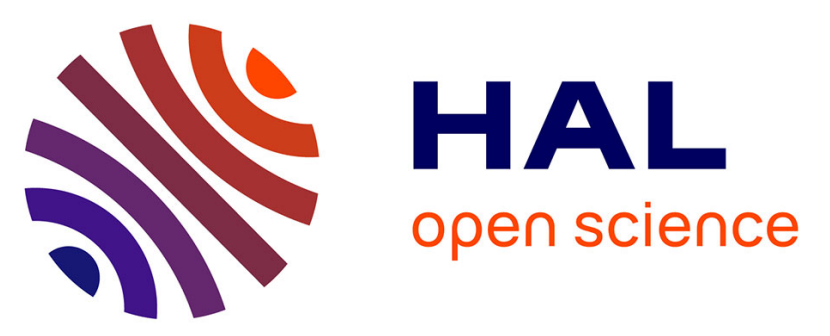

\title{
Constraining Ion-Scale Heating and Spectral Energy Transfer in Observations of Plasma Turbulence
}

\author{
T.A. Bowen, A. Mallet, S.D. Bale, J.W. Bonnell, A.W. Case, B.D.G.
}

Chandran, A. Chasapis, C.H.K. Chen, D. Duan, Thierry Dudok de Wit, et al.

\section{- To cite this version:}

T.A. Bowen, A. Mallet, S.D. Bale, J.W. Bonnell, A.W. Case, et al.. Constraining Ion-Scale Heating and Spectral Energy Transfer in Observations of Plasma Turbulence. Physical Review Letters, 2020, 125 (2), 8 p. 10.1103/PhysRevLett.125.025102 . insu-02923463

HAL Id: insu-02923463

https://hal-insu.archives-ouvertes.fr/insu-02923463

Submitted on 27 Aug 2020

HAL is a multi-disciplinary open access archive for the deposit and dissemination of scientific research documents, whether they are published or not. The documents may come from teaching and research institutions in France or abroad, or from public or private research centers.
L'archive ouverte pluridisciplinaire HAL, est destinée au dépôt et à la diffusion de documents scientifiques de niveau recherche, publiés ou non, émanant des établissements d'enseignement et de recherche français ou étrangers, des laboratoires publics ou privés. 


\title{
Constraining Ion-Scale Heating and Spectral Energy Transfer in Observations of Plasma Turbulence
}

\author{
Trevor A. Bowen $\odot,{ }^{1, *}$ Alfred Mallet $\odot,{ }^{1}$ Stuart D. Bale $\odot,{ }^{1,2,3,4}$ J. W. Bonnell $\odot,{ }^{1}$ Anthony W. Case $\odot,{ }^{5}$ \\ Benjamin D. G. Chandran $\odot,{ }^{6,7}$ Alexandros Chasapis $\odot,{ }^{8}$ Christopher H. K. Chen $\odot,{ }^{4}$ Die Duan $\odot,{ }^{1,9}$ \\ Thierry Dudok de Wit $\odot,{ }^{10}$ Keith Goetz $\odot,{ }^{11}$ Jasper S. Halekas $\odot,{ }^{12}$ Peter R. Harvey $\odot,{ }^{1}$ J. C. Kasper $\odot,{ }^{13,5}$ \\ Kelly E. Korreck $\odot,{ }^{5}$ Davin Larson $\odot,{ }^{1}$ Roberto Livi $\odot,{ }^{1}$ Robert J. MacDowall $\odot,{ }^{14}$ David M. Malaspina $\odot,{ }^{15,8}$ Michael \\ D. McManus $\odot,{ }^{1,2}$ Marc Pulupa $\odot,{ }^{1}$ Michael Stevens $\odot,{ }^{5}$ and Phyllis Whittlesey $\oplus^{1}$ \\ ${ }^{1}$ Space Sciences Laboratory, University of California, Berkeley, California 94720-7450, USA \\ ${ }^{2}$ Physics Department, University of California, Berkeley, California 94720-7300, USA \\ ${ }^{3}$ The Blackett Laboratory, Imperial College London, London SW7 2AZ, United Kingdom \\ ${ }^{4}$ School of Physics and Astronomy, Queen Mary University of London, London E1 4NS, United Kingdom \\ ${ }^{5}$ Smithsonian Astrophysical Observatory, Cambridge, Massachusetts 02138, USA \\ ${ }^{6}$ Department of Physics and Astronomy, University of New Hampshire, Durham, New Hampshire 03824, USA \\ ${ }^{7}$ Space Science Center, University of New Hampshire, Durham, New Hampshire 03824, USA \\ ${ }^{8}$ Laboratory for Atmospheric and Space Physics, University of Colorado, Boulder, Colorado 80303, USA \\ ${ }^{9}$ School of Earth and Space Sciences, Peking University, Beijing 100871, China \\ ${ }^{10}$ LPC2E, CNRS and University of Orléans, 3 Avenue de la Recherche Scientifique, 45071 Orléans, France \\ ${ }^{11}$ School of Physics and Astronomy, University of Minnesota, Minneapolis, Minnesota 55455, USA \\ ${ }^{12}$ Department of Physics and Astronomy, University of Iowa, Iowa City, Iowa 52242, USA \\ ${ }^{13}$ Climate and Space Sciences and Engineering, University of Michigan, Ann Arbor, Michigan 48109, USA \\ ${ }^{14}$ Solar System Exploration Division, NASA/Goddard Space Flight Center, Greenbelt, Maryland 20771, USA \\ ${ }^{15}$ Astrophysical and Planetary Sciences Department, University of Colorado, Boulder, Colorado, USA
}

(Received 14 January 2020; revised 11 May 2020; accepted 22 May 2020; published 6 July 2020)

\begin{abstract}
We perform a statistical study of the turbulent power spectrum at inertial and kinetic scales observed during the first perihelion encounter of the Parker Solar Probe. We find that often there is an extremely steep scaling range of the power spectrum just above the ion-kinetic scales, similar to prior observations at 1 A.U., with a power-law index of around -4. Based on our measurements, we demonstrate that either a significant $(>50 \%)$ fraction of the total turbulent energy flux is dissipated in this range of scales, or the characteristic nonlinear interaction time of the turbulence decreases dramatically from the expectation based solely on the dispersive nature of nonlinearly interacting kinetic Alfvén waves.
\end{abstract}

DOI: 10.1103/PhysRevLett.125.025102

Introduction.-In many astrophysical settings, the background plasma is highly turbulent and nearly collisionless. The dissipation of collisionless turbulence is important for plasma heating [1-5], but the precise mechanisms involved are a matter of debate [6]. The solar wind provides a convenient example of a collisionless plasma in which both large magnetohydrodynamic scales (MHD) and subion-kinetic scales can be studied through in situ spacecraft measurements.

The observed ion temperature profiles in the solar wind require significant perpendicular ion heating [7], which is likely initiated at ion-kinetic scales, where particles interact efficiently with electromagnetic waves [1,8-14]. Such heating should transfer energy from the waves to the particles, generating observable electromagnetic signatures. The observation of spectral steepening in the "transition range" between MHD and ion-kinetic scales is most certainly a result of these processes [15-19].
Large-scale fluctuations appear to be nonlinearly interacting Alfvénic turbulence [20], with a power-law spectrum between $k_{\perp}^{-5 / 3}[21]$ and $k_{\perp}^{-3 / 2}[22,23]$, in rough agreement with various MHD turbulence theories [24-27]. At scales much smaller than the ion gyroradius $\rho_{i}=v_{\text {thi }} / \Omega_{i}$ (with $v_{\text {thi }}=\sqrt{2 T_{0 i} / m_{i}}$ the ion thermal speed and $\Omega_{i}=Z e B_{0} / m_{i}$ the ion gyrofrequency), the spectrum steepens to about $k_{\perp}^{-2.8}$ [28-31], as nondispersive Alfvénic turbulence transitions to dispersive kinetic Alfvénic turbulence [32]. This steepening occurs solely due to the dispersion relation, without any dissipation: adiabatic fluid approximation to the dynamics in this range of scales predicts a $k_{\perp}^{-7 / 3}$ spectrum $[13,33]$. Simulations in the fluid approximation obtain the slightly steeper $k_{\perp}^{-8 / 3}$ spectrum [34], with the difference ascribed to intermittency. Fully kinetic models recover similar scalings [35-37].

At ion scales, i.e., the transition from MHD to kinetic regimes with $k_{\perp} \rho_{i} \sim 1$, a spectrum significantly steeper 
than the sub-ion $k_{\perp}^{-2.8}$ scaling has been reported $[9,15,17$, 19,38-40]. Instrumental limitations initially led to interpretation of ion-scale steepening as variability in the kinetic range (i.e., $k \rho_{i}>1$ ) spectrum, rather than a signature of processes localized to ion scales [9,38,39]. Measurements of fluctuations deep in the sub-ion scales repeatedly demonstrate a $k_{\perp}^{-2.8}$ spectra, indicating that transition range steepening disappears at scales $k_{\perp} \rho>1 \quad$ [28-31]. Observational signatures of the transition range are intermittent, nonregular, and variable and cannot be explained by the kinetic Alfvén wave (KAW) dispersion relation [17,18,38,39,41]. Two proposed explanations for transition-range steepening are, first, strong dissipation of the turbulence around the ion scales [15-18,42]. Second, nonlinear effects that increase turbulent cascade rates: e.g., the onset of reconnection [43-45], the increasing importance of nonlinear interactions between copropagating waves [46], or the influence of coherent structures $[40,47,48]$.

In this Letter, we report Parker Solar Probe (PSP) observations of strong and regular ion-scale steepening in the inner heliosphere, indicating that a dispersive transition from nonlinear Alfvén turbulence at MHD scales to kinetic Alfvén turbulence at sub-ion scales is an incomplete description of solar wind turbulence. Increased levels of ion-scale steepening in PSP observations enable a comprehensive statistical study of the phenomenon. Using continuity of turbulent energy flux, assuming local wavenumber spectral transfer $[12,13,49,50]$, we demonstrate that the observed steepening has drastic implications for turbulent heating and/or nonlinear cascade rates. Statistical analysis shows that transition-range steepening is consistent with levels of dissipation sufficient for required bulk in situ heating of the solar wind [14,51-56]. As our model cannot identify heating as the unique cause of the steepening, we consider an alternative framework, in which steepening is the result of increased spectral transfer rates. Though our analysis is largely inconsistent with increased spectral transfer via imbalanced turbulence or magnetic reconnection $[43,44,46]$, we cannot explicitly exclude other nonlinear dynamics that may anomalously accelerate turbulent energy transfer at ion scales.

Cascade model.-We use a Batchelor cascade model $[13,49]$, which relates turbulent energy flux $\epsilon_{k_{\perp}}$ through wave-number $k_{\perp}$ to the spectrum $E_{k_{\perp}}=b_{k_{\perp}}^{2} / k_{\perp}$, where $b_{k_{\perp}}$ is the turbulent amplitude, through

$$
\epsilon_{k_{\perp}}=\omega_{c} k_{\perp} E_{k_{\perp}} .
$$

The quantity

$$
\omega_{c}=k_{\perp} b_{k_{\perp}} \tilde{\omega}_{k_{\perp}}=k_{\perp}^{3 / 2} E_{k_{\perp}}^{1 / 2} \tilde{\omega}_{k_{\perp}}
$$

is the characteristic spectral transfer rate (inverse cascade time) at $k_{\perp}$, and $\tilde{\omega}_{k_{\perp}}$ parametrizes both dispersive (e.g., from the dispersive KAW [33]) and/or nonlinear effects (e.g., caused by dynamic alignment [25], intermittency [34], or reconnection [43]). All unknown normalizing constants are contained in $\tilde{\omega}_{k_{\perp}}$. In statistical steady state far from the injection scales $[13,57]$ one obtains a simple equation with solution

$$
1-\hat{Q}_{k_{1}, k_{2}}=\frac{\epsilon_{k_{2}}}{\epsilon_{k_{1}}}=\exp \left\{-\int_{k_{1}}^{k_{2}} \frac{\gamma_{k_{\perp}}}{\omega_{c}} \frac{d k_{\perp}}{k_{\perp}}\right\},
$$

where $\gamma_{k_{\perp}}$ is the energy dissipation rate at $k_{\perp}$, and $\hat{Q}_{k_{1}, k_{2}}$ is the fractional heating rate over the range $\left[k_{1}, k_{2}\right]$. If $\gamma_{k_{\perp}}=0$ over $\left[k_{1}, k_{2}\right]$, then $\epsilon_{k_{\perp}}$ is constant over $\left[k_{1}, k_{2}\right]$. In this Letter, we do not assume a physical mechanism for dissipation, rather we use empirical observations of spectral steepening to constrain required levels of dissipation. However, the functional form of $\gamma_{k_{\perp}}$ has significant impact on the form of the energy spectrum [12,13,17,31,58,59]. Using Eqs. (1) and (2),

$$
\frac{E_{k_{2}}}{E_{k_{1}}}=\left(\frac{k_{2}}{k_{1}}\right)^{-5 / 3}\left(\frac{\epsilon_{k_{2}}}{\epsilon_{k_{1}}}\right)^{2 / 3}\left(\frac{\tilde{\omega}_{k_{1}}}{\tilde{\omega}_{k_{2}}}\right)^{2 / 3} .
$$

Equation (4) relates in situ measurements of $E_{k}$ to the turbulent heating rate (second bracket) and/or anomalous dispersive and nonlinear effects (third bracket). Surveying transition-range steepening in $E_{k}$ illustrates the dramatic effect that it takes on the turbulent cascade.

Data and fitting.-The PSP mission [60] provides a set of in situ measurements [61,62] enabling detailed studies of turbulence in the inner heliosphere [23, 63-65]. Preliminary observations show a steep $f^{-3}-f^{-4}$ spectrum of the magnetic field fluctuations at ion scales [66-68], but instrumental sensitivity has precluded further detailed study.

We use merged fluxgate and search coil measurements (SCM) from PSP FIELDS [61,69] operating at $293 \mathrm{~Hz}$, enabling measurement of inertial, transition, and kinetic scales. We study the first PSP perihelion encounter, using the interval with maximal data rates: November 4, 2018, 09:28:19-November 7, 2018, 09:28:19. During this period, PSP was magnetically connected to a small equatorial coronal hole generating slow, but highly Alfvénic solar wind $[66,70]$.

We partition the interval into an ensemble of $2^{16}$ samples ( 223.69 s), with $50 \%$ overlap to improve statistics. Intervals were rejected for lack of finite measurements, or if the SCM was in low gain mode. Intervals with ionscale waves, which strongly affect measurements of ionscale turbulence, are excluded [40,66,71]. In total, 243 intervals were kept. We consider frequencies up to $100 \mathrm{~Hz}$ $\left(k_{\perp} \rho_{i} \sim 10\right)$, to avoid the SCM noise floor. Average plasma properties are computed for each interval using solar wind electrons alphas and protons (SWEAP) investigation data [62]; $n_{i}$ and $T_{i}$ from the SWEAP-solar probe cup (SPC) [72] and $n_{e}, T_{e}$ from SWEAP-solar probe analyzers 

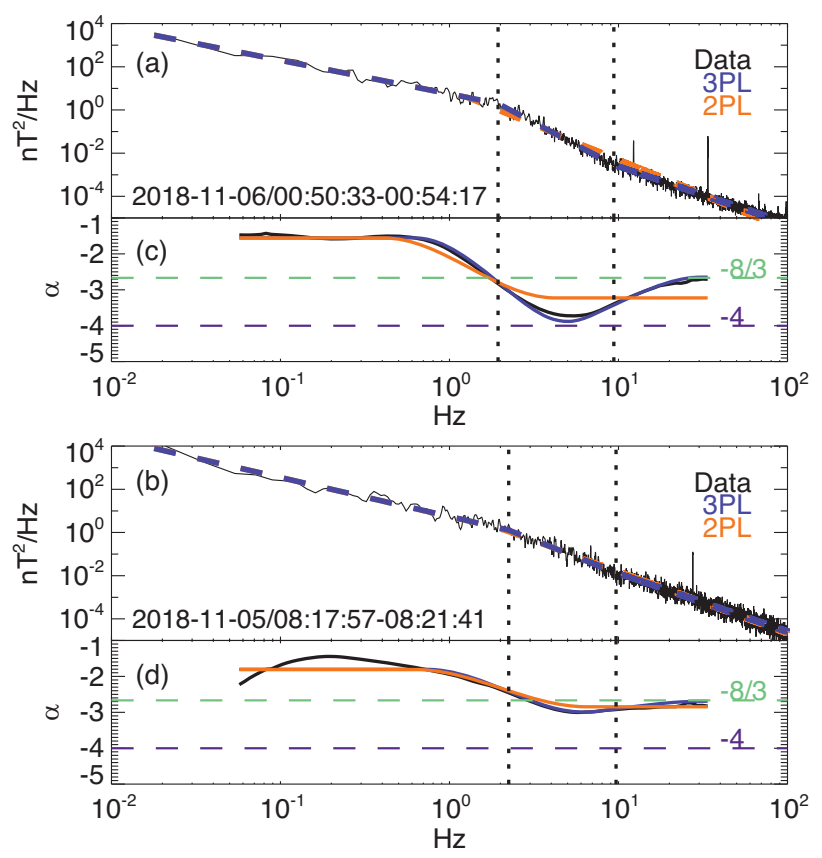

FIG. 1. (a,b) Examples of PSP/FIELDS magnetic field spectra with 3PL (blue) and 2PL fits (orange). Vertical lines show 3PL spectral breaks. (c,d) spectral indices for data (black), 3PL (blue) and 2PL fits (orange). Horizontal lines are shown corresponding to spectral indices of $-8 / 3$ (teal) and -4 (purple). Top interval has statistically significant spectral steepening, while the bottom interval is sufficiently fit by $2 \mathrm{PL}$.

(SPAN) electron fits $[73,74]$. On average, $\beta_{i}\left(\beta_{e}\right)$ is 0.26 (0.74) with a standard deviation of $0.13(0.25)$, with $\beta_{i}=$ $v_{\text {thi }}^{2} / v_{A}^{2}$ and the Alfvén speed is $v_{A}=B_{0} / \sqrt{n_{0 i} m_{i} \mu_{0}}$. The average $T_{0 i} / T_{0 e}$ is 0.5 . The mean normalized cross helicity $\sigma_{c} \sim 0.75$.

KAWs have intrinsic density fluctuations [33,75-78], which at $k_{\perp} \rho_{i} \gtrsim 1$ provide a non-negligible contribution to the total energy. We estimate this contribution by determining $\delta n_{e}$ from the pressure balance $\delta B_{\|} / B_{0}=-\left(\beta_{i} / 2\right)(1+$ $\left.Z T_{0 e} / T_{0 i}\right) \delta n_{e} / n_{0 e}$, appropriate for KAWs, and estimate the total energy as

$$
E_{\mathrm{tot}}=\frac{|\delta \mathbf{B}|^{2}}{2 \mu_{0}}+\frac{n_{0 e} T_{0 e}}{2}\left(\frac{\delta n_{e}}{n_{0}}\right)^{2} .
$$

Figure 1(a) shows an example with transition-range steepening to an $\sim f^{-4}$ spectrum at ion scales, similar to observations at 1 A.U. [17,19]. At the highest frequencies, the measured spectral index is consistent with kinetic Alfvén wave scaling of $E \propto f^{-8 / 3}$. Transition-range steepening is not obvious in a second example interval, Fig. 1(b), but approximate $f^{-8 / 3}$ scaling is evident.

Continuous power-law (PL) functions with two and three spectral ranges (2PL, 3PL) are fit to each interval, optimizing $\chi^{2}$ residuals. Figures $1(\mathrm{c})$ and $1(\mathrm{~d})$ show the variation of spectral index over scales for the data and two models.
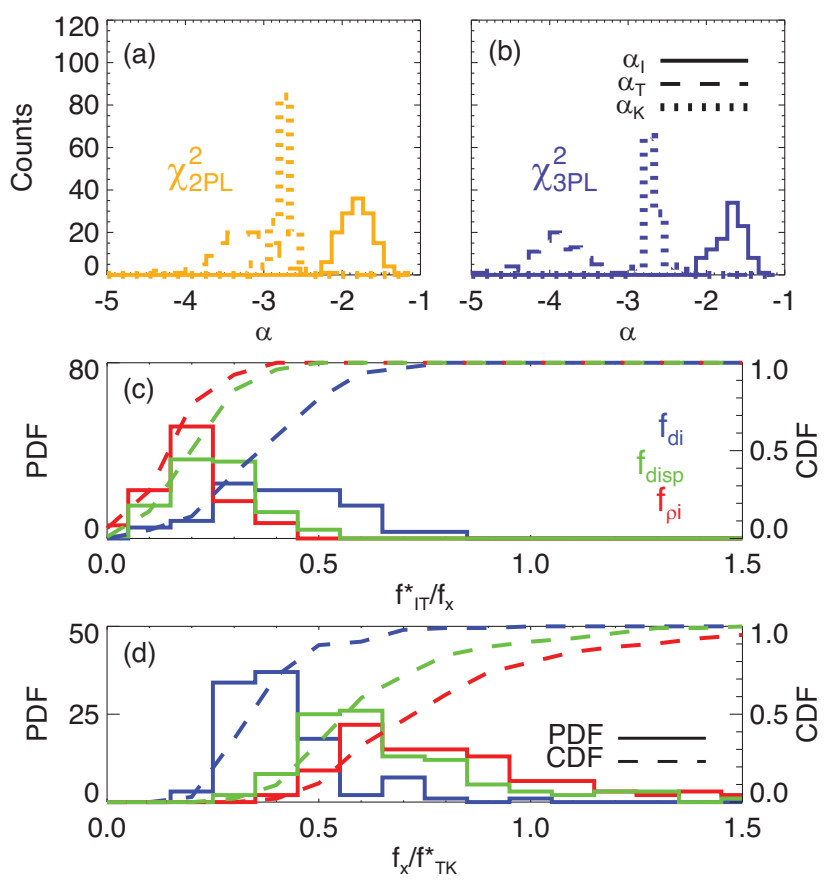

FIG. 2. (a) Probability distribution function (PDF) of fitted 3PL spectral indices for the $\chi_{2 \mathrm{PL}}^{2}$ (orange) population for inertial $\alpha_{I}$ (solid), transition $\alpha_{T}$ (dashed), and kinetic $\alpha_{K}$ (dotted) ranges. (b) PDF of fitted 3PL spectral indices for the $\chi_{3 \mathrm{PL}}^{2}$ (blue) population. (c) PDF and cumulative distribution function (CDF) of $f_{I T}^{*}$ normalized to spacecraft frequencies $f_{x}=$ $\left(f_{\rho i}, f_{d i}, f_{\text {disp }}\right)$ (red, blue, green). (d) PDF and CDF of $f_{T K}^{*}$ normalized to ion scales $\left(f_{x} / f_{T K}^{*}\right)$. Normalizations in (c) and (d) show $f_{I T}^{*} / f_{x}<1$ and $f_{T K}^{*} / f_{x}<1$, indicating ion scales are within the transition range.

The 3PL fit determines spectral indices of the inertial $\left(\alpha_{I}\right)$, transition, $\left(\alpha_{T}\right)$, and kinetic $\left(\alpha_{K}\right)$ ranges and the break points $\left(f_{I T}^{*}\right.$ and $\left.f_{T K}^{*}\right)$. The significance of including a third spectral range, which introduces additional degrees of freedom $\left(f_{I T}^{*}\right.$ and $\left.\alpha_{T}\right)$, is determined by the statistical $f$ test. Though not shown, a continuum of significance values is measured, indicating that, at times, spectral steepening is strong (i.e., the 3PL fit significantly improves $\chi^{2}$ ), whereas some intervals have moderate or no statistically significant steepening (i.e., $\chi^{2}$ is similar for both 3PL and 2PL fits). As a continuous distribution of spectral steepening is measured, intervals are separated into two equal sized populations, approximately distinguishing spectra well fit by $3 \mathrm{PL}$ from those sufficiently described by $2 \mathrm{PL}$. The notation $\chi_{3 \mathrm{PL}}^{2}$ and $\chi_{2 \mathrm{PL}}^{2}$ refers to the populations.

Figures 2(b) and 2(c) show the histogram of measured spectral indices for 3PL fits for $\alpha_{I}, \alpha_{T}$, and $\alpha_{K}$. Figures 2(b) and 2(c) show distributions for the $\chi_{2 \mathrm{PL}}^{2}$ and $\chi_{3 \mathrm{PL}}^{2}$ populations. Mean spectral indices for each range and population are in Table I. The total population has a mean transitionrange index of $\left\langle\alpha_{T}\right\rangle=-3.5$, while intervals with most significant steepening have $\left\langle\alpha_{T}^{\chi_{3 \mathrm{PL}}^{2}}\right\rangle=-3.9$. 
TABLE I. Measured mean spectral indices from 3PL and 2PL fits to both $\chi_{2 \mathrm{PL}}^{2}$ and $\chi_{3 \mathrm{PL}}^{2}$ populations.

\begin{tabular}{lcccccc}
\hline \hline & \multicolumn{2}{c}{ 2PL fit } & & \multicolumn{3}{c}{ 3PL fit } \\
\cline { 2 - 3 } \cline { 5 - 7 } Population & $\left\langle\alpha_{I}\right\rangle$ & $\left\langle\alpha_{K}\right\rangle$ & & $\left\langle\alpha_{I}\right\rangle$ & $\left\langle\alpha_{T}\right\rangle$ & $\left\langle\alpha_{K}\right\rangle$ \\
\hline$\chi_{2 \mathrm{PL}}^{2}$ & -1.7 & -2.9 & & -1.7 & -3.2 & -2.7 \\
$\chi_{3 \mathrm{PL}}^{2}$ & -1.6 & -3.1 & & -1.6 & -3.9 & -2.6 \\
$\chi_{3 \mathrm{PL}}^{2} \cup \chi_{2 \mathrm{PL}}^{2}$ & -1.6 & -3.0 & & -1.7 & -3.5 & -2.7 \\
\hline \hline
\end{tabular}

The spacecraft frequencies $f_{\rho_{i}}, f_{d_{i}}$, and $f_{\rho_{\text {disp }}}$ associated with the ion gyroscale $\rho_{i}$, inertial scale $d_{i}=\rho_{i} / \sqrt{\beta_{i}}$, and the scale at which dispersion sets in $\rho_{\text {disp }}=\rho_{i} \sqrt{\left(1+T_{0 e} / T_{0 i}\right) / 2}$, are computed using the Taylor hypothesis $2 \pi f=v_{s w} k$. Figures 2(d) and 2(e) show distributions of the break scales of the $\chi_{3 \mathrm{PL}}^{2}$ population normalized to the frequencies $f_{\rho_{i}}, f_{d_{i}}$, and $f_{\rho_{\text {disp }}}$. Figure 2(d) shows that the break from the inertial to transition range occurs at frequencies significantly lower than ion scales.

Physical interpretation: Dissipation.-Equation (4) implies that a steep transition range is associated with either significant dissipation or nonlinear speedup of the cascade. First, we assume that the steep spectrum is due to dissipation $[8,15,57,59,79-81]$; i.e., $\epsilon_{k_{\perp}}$ decreases with $k_{\perp}$ across the transition range. Using the $3 \mathrm{PL}$ fits, we construct a synthetic spectrum

$$
E^{\prime}(f)= \begin{cases}c_{I} f^{\alpha_{I}} & \text { if } f<f_{I T}^{*} \\ c_{T} f^{\alpha_{K}} & \text { if } f>f_{I T}^{*}\end{cases}
$$

This joins the fitted inertial-range spectrum to a synthetic spectrum with the fitted kinetic-range exponent $\alpha_{k}$ at the inertial-transition break $f_{I T}^{*}$ An example of this synthetic spectrum is shown in Fig. 3(a). We use $E^{\prime}$ to determine $\tilde{\omega}_{k_{\perp}}^{\prime}$ [cf. Eqs. (1) and (2)], assuming that this synthetic spectrum is the result of constant $\epsilon_{k_{\perp}}^{\prime}$. Using this synthetic $\tilde{\omega}_{k_{\perp}}^{\prime}$ and the fitted 3PL spectrum $E^{3 \mathrm{PL}}$ in Eq. (4) results in an estimate of the heating in the transition range relative to the synthetic spectrum,

$$
1-\hat{Q}^{*}=\frac{\epsilon_{T K}}{\epsilon_{I T}}=\left(\frac{E^{3 \mathrm{PL}}\left(f_{T K}^{*}\right)}{E^{\prime}\left(f_{T K}^{*}\right)}\right)^{3 / 2} .
$$

Figure 3(b) shows measured ratios of $\epsilon_{T K} / \epsilon_{I T}$ as a function of transition-range spectral index $\alpha_{T}$. Transitionrange spectral indices of $\alpha_{T} \approx-3.5$ correspond to heating at $\hat{Q}^{*}>50 \%$ of the turbulent energy flux and thus may be a signature of significant ion-scale heating.

Physical interpretation: Nonlinear effects.-The steep transition-range spectrum is alternatively explained by nonlinear effects that increase nonlinear spectral transfer rates. Assuming that $\epsilon_{k_{\perp}}$ is constant, we use Eq. (4) to determine the scaling of $\tilde{\tilde{\omega}}_{k_{\perp}}$, and therefore $\omega_{c}$, that matches each interval's measured spectrum. Figure 3(c) shows the scaling of $\omega_{c}$ corresponding to the interval in Fig. 1(a). In the inertial range, the wave-number power-law scalings, $\omega_{c} \propto k_{\perp}^{\alpha_{\omega}}$, are similar to those predicted in MHD turbulence models: between $\omega_{c} \propto k_{\perp}^{2 / 3}$ [24] and $\omega_{c} \propto k_{\perp}^{1 / 2}$ [25]. In the kinetic range, the scaling is similar to predictions of the KAW turbulence models: between $\omega_{c} \propto k_{\perp}^{4 / 3}$ [33] and $\omega_{c} \propto$ $k_{\perp}^{5 / 3}$ [34]. The scaling of $\omega_{c}$ is significantly steeper in the ion-scale transition.

The fitted $E^{3 \mathrm{PL}}$ and synthetic $E^{\prime}$ spectra allow an estimate of the increase in nonlinear interactions due to transitionrange steepening, using Eq. (1) and taking $\epsilon_{k_{\perp}}$ constant.
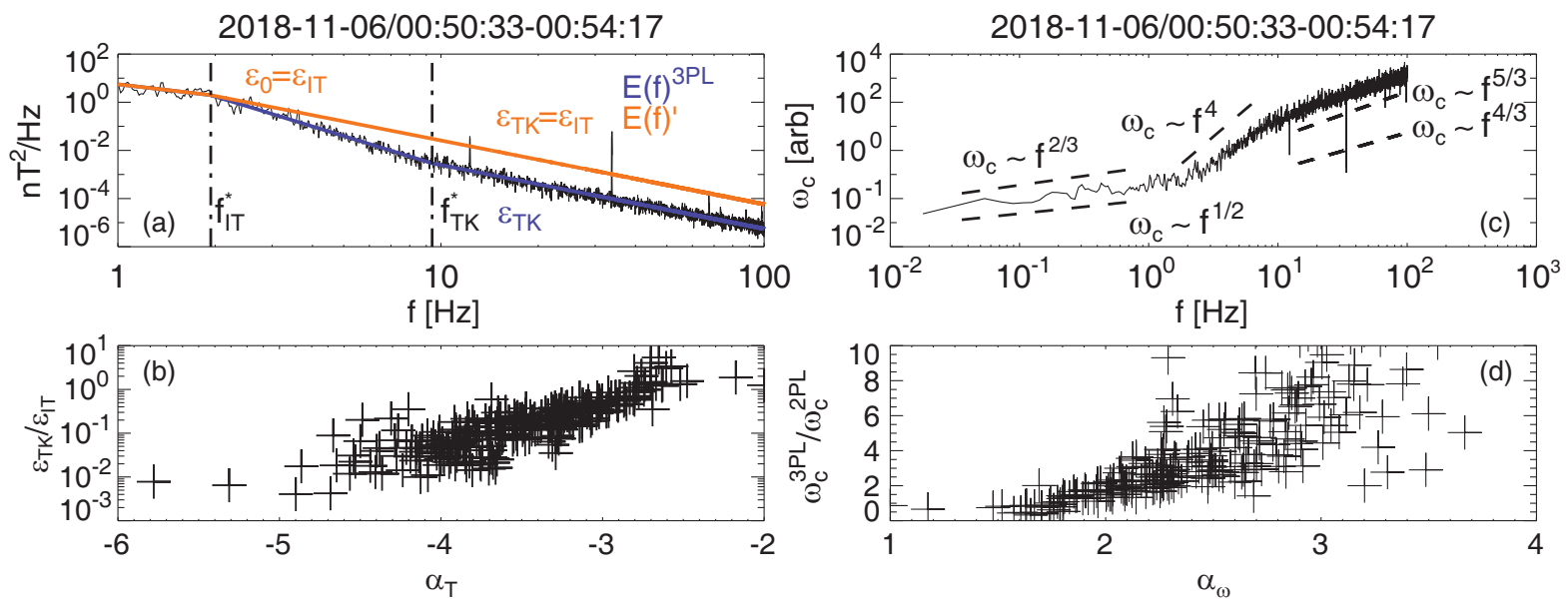

FIG. 3. (a) Measured power spectrum with transition-range steepening from Fig. 1(a) (black), with the corresponding fitted (blue) and synthetic (orange) spectra. Fit break scales are plotted (vertical lines). (b) Measured ratios of energy flux at transition-kinetic break relative to inertial-kinetic break scale $\epsilon_{T K} / \epsilon_{I T}$, plotted against transition-range spectral index $\alpha_{T}$. (c) Nonlinear frequency $\omega_{c}$ as function of $f$, assuming $\epsilon_{k_{\perp}}$ at all frequencies. Various power-law scalings of $\omega_{c} \sim f^{\alpha_{\omega}}$ are shown. (d) Increase of $\omega_{c}$ over the transition range relative to the synthetic spectrum as a function of $\alpha_{\omega}$ for all intervals. 
Figure 3(d) shows the ratio of $\omega_{c}^{3 \mathrm{PL}} / \omega_{c}^{\prime}$ evaluated at $f_{T K}^{*}$. Evidently, for nonlinear effects to explain the steeper spectra without dissipation, $\omega_{c}$ must anomalously increase by a large factor of $\gtrsim 5$.

Discussion.-We have performed a statistical study of the scaling properties of the turbulent fluctuation spectrum in the inner heliosphere using data from the first PSP encounter. At low frequencies, there is an "inertial range" with a spectral index ranging from $-5 / 3$ to $-3 / 2$ [21-23], while at high frequencies, there is a "kinetic range" with a spectral index of around -2.7 [28-30]. Between these, an anomalously steep transition range with a highly variable spectrum typically appears [15-19]. Even in intervals where transition-range steepening is not particularly dramatic, spectra at ion scales $\left(k_{\perp} \rho \sim 1\right)$ remain steeper than the deep sub-ion-range spectrum $\left(k_{\perp} \rho>1\right)$. This indicates that three spectral ranges are regularly necessary to describe collisionless plasma turbulence throughout the inertial and ion-kinetic ranges.

Ion-scale steepening either corresponds to significant turbulent dissipation, or a dramatic change in nonlinear cascade rates. Using synthetic spectra with no ion-scale steepening, we empirically constrain the effects associated with steepening. If dissipation is the dominant effect, the typically observed transition-range spectrum of -3.5 corresponds roughly to a $50 \%$ dissipation of energy flux in this range, indicating significant ion-scale heating. The half of intervals with the strongest signatures of ion-scale steepening have a typical transition index of -3.9 , corresponding to heating rates that are $\gtrsim 90 \%$ of the turbulent energy flux. This amount of heating is consistent with the turbulent dissipation required to power the solar wind [14,51-56]. Measuring the radial trends in transition-range heating may account for solar wind temperature profile and heating rates at 1 A.U.

Several competing mechanisms have been suggested for ion-scale dissipation: e.g., stochastic heating [42], Landau damping [13,17,76,77], and cyclotron resonance $[10,16,18]$. Correlations between signatures of the transition range and parameters associated with stochastic heating were not found $[42,63]$. Measurements of 3D ion distributions will enable comparison of damping rates associated with, e.g., Landau and cyclotron resonance, with our observed dissipation rates [1,8,10-14,17,18,76,82,83]. Additionally, heating processes may leave clear signatures in the distribution function: e.g., diffusive shells in the case of cyclotron resonance [84] or flattening associated with resonant damping or perpendicular stochastic heating $[63,85]$. Use of electric fields will enable analysis of alignment over the transition range, enabling further constraint of energy transfer, heating rates, and mechanisms [86-88].

The Batchelor cascade model (3) and (4) cannot uniquely distinguish heating from increased spectral transfer rates. If anomalous variations in cascade rates are responsible for ion-scale steepening, then the nonlinear frequency (inverse cascade time) of the turbulence must increase by a factor of $\gtrsim 5$ relative to unsteepened intervals. One possibility for increased nonlinear transfer includes reconnection onset and loss of dynamic alignment [43-45]; however, we find no connection between the critical parameters for reconnection and the transition range. Additionally, nonlinear interactions between copropagating waves in imbalanced turbulence may cause ion-scale steepening [46]. However, the required imbalance is significantly larger than what is observed in the inner heliosphere $[64,65]$. No correlation between spectral steepening and cross helicity was found, throwing the influence of alignment on ion-scale steepening further into doubt. Effects of damping on highly imbalanced turbulence may provide insight into the transition range [89]. Though we exclude intervals with coherent waves, the presence of intermittency may affect nonlinear interaction at ion scales $[40,47,48,50,90]$. Resolving such effects in PSP likely requires electric field measurements to constrain turbulent imbalance and density fluctuations at faster cadences than currently available.

We demonstrate that ion-scale spectral steepening is a dramatic effect in collisionless plasma turbulence. While further work is needed to distinguish exact mechanisms behind observed ion-scale spectral steepening, this Letter largely constrains their effects on the turbulent cascade.

The FIELDS and SWEAP instruments were designed and built under NASA Contract No. NNN06AA01C. S. D. B. acknowledges the support of the Leverhulme Trust Visiting Professorship program. C. H. K. C. is supported by STFC Ernest Rutherford Fellowship ST/N003748/2. B. D. G. C. was supported in part by NASA Grants No. NNX17AI18G and No. 80NSSC19K0829. D. D. is supported by the China Scholarship Council for his stay at SSL.

*tbowen@berkeley.edu

[1] E. Quataert, Particle heating by Alfvénic turbulence in hot accretion flows, Astrophys. J. 500, 978 (1998).

[2] S. R. Cranmer, Ion cyclotron wave dissipation in the solar corona: The summed effect of more than 2000 ion species, Astrophys. J. 532, 1197 (2000).

[3] S. R. Cranmer and A. A. van Ballegooijen, Alfvénic turbulence in the extended solar corona: Kinetic effects and proton heating, Astrophys. J. 594, 573 (2003).

[4] I. Zhuravleva, E. Churazov, A. A. Schekochihin, S. W. Allen, P. Arévalo, A. C. Fabian, W. R. Forman, J. S. Sanders, A. Simionescu, R. Sunyaev, A. Vikhlinin, and N. Werner, Turbulent heating in galaxy clusters brightest in $\mathrm{x}$-rays, Nature (London) 515, 85 (2014).

[5] C. H. K. Chen, K. G. Klein, and G. G. Howes, Evidence for electron Landau damping in space plasma turbulence, Nat. Commun. 10, 740 (2019).

[6] T. N. Parashar, C. Salem, R. T. Wicks, H. Karimabadi, S. P. Gary, and W. H. Matthaeus, Turbulent dissipation challenge: 
A community-driven effort, J. Plasma Phys. 81, 905810513 (2015).

[7] J. D. Richardson, K. I. Paularena, A. J. Lazarus, and J. W. Belcher, Radial evolution of the solar wind from IMP 8 to Voyager 2, Geophys. Res. Lett. 22, 325 (1995).

[8] E. Quataert and A. Gruzinov, Turbulence and particle heating in advection-dominated accretion flows, Astrophys. J. 520, 248 (1999).

[9] R. J. Leamon, C. W. Smith, N. F. Ness, W. H. Matthaeus, and H. K. Wong, Observational constraints on the dynamics of the interplanetary magnetic field dissipation range, J. Geophys. Res. 103, 4775 (1998).

[10] R. J. Leamon, W. H. Matthaeus, C. W. Smith, and H. K. Wong, Contribution of cyclotron-resonant damping to kinetic dissipation of interplanetary turbulence, Astrophys. J. Lett. 507, L181 (1998).

[11] S. Peter Gary, Collisionless dissipation wavenumber: Linear theory, J. Geophys. Res. 104, 6759 (1999).

[12] R. J. Leamon, W. H. Matthaeus, C. W. Smith, G. P. Zank, D. J. Mullan, and S. Oughton, MHD-driven kinetic dissipation in the solar wind and corona, Astrophys. J. 537, 1054 (2000).

[13] G. G. Howes, S. C. Cowley, W. Dorland, G. W. Hammett, E. Quataert, and A. A. Schekochihin, A model of turbulence in magnetized plasmas: Implications for the dissipation range in the solar wind, J. Geophys. Res. 113, A05103 (2008).

[14] B. D. G. Chandran, T. J. Dennis, E. Quataert, and S. D. Bale, Incorporating kinetic physics into a two-fluid solar-wind model with temperature anisotropy and low-frequency Alfvén-wave turbulence, Astrophys. J. 743, 197 (2011).

[15] K. U. Denskat, H. J. Beinroth, and F. M. Neubauer, Interplanetary magnetic field power spectra with frequencies from $2.4 \times 10^{-5} \mathrm{~Hz}$ to $470 \mathrm{~Hz}$ from HELIOS-observations during solar minimum conditions, J. Geophys. 54, 60 (1983), https://ui.adsabs.harvard.edu/abs/1983JGZG...54.. .60D/abstract.

[16] S. A. Markovskii, B. J. Vasquez, C. W. Smith, and J. V. Hollweg, Dissipation of the perpendicular turbulent cascade in the solar wind, Astrophys. J. 639, 1177 (2006).

[17] F. Sahraoui, M. L. Goldstein, G. Belmont, P. Canu, and L. Rezeau, Three Dimensional Anisotropic $k$ Spectra of Turbulence at Subproton Scales in the Solar Wind, Phys. Rev. Lett. 105, 131101 (2010).

[18] C. W. Smith, B. J. Vasquez, and J. V. Hollweg, Observational constraints on the role of cyclotron damping and kinetic Alfvén waves in the solar wind, Astrophys. J. 745, 8 (2012).

[19] K. H. Kiyani, K. T. Osman, and S. C. Chapman, Dissipation and heating in solar wind turbulence: From the macro to the micro and back again, Phil. Trans. R. Soc. A 373, 20140155 (2015).

[20] C. H. K. Chen, Recent progress in astrophysical plasma turbulence from solar wind observations, J. Plasma Phys. 82, 535820602 (2016).

[21] W. H. Matthaeus and M. L. Goldstein, Measurement of the rugged invariants of magnetohydrodynamic turbulence in the solar wind, J. Geophys. Res. 87, 6011 (1982).

[22] J. J. Podesta, D. A. Roberts, and M. L. Goldstein, Spectral exponents of kinetic and magnetic energy spectra in solar wind turbulence, Astrophys. J. 664, 543 (2007).
[23] C. H. K. Chen et al., The evolution and role of solar wind turbulence in the inner heliosphere, Astrophys. J. Suppl. Ser. 246, 53 (2020).

[24] P. Goldreich and S. Sridhar, Toward a theory of interstellar turbulence. II. Strong Alfvénic turbulence, Astrophys. J. 438, 763 (1995).

[25] S. Boldyrev, Spectrum of Magnetohydrodynamic Turbulence, Phys. Rev. Lett. 96, 115002 (2006).

[26] B. D. G. Chandran, A. A. Schekochihin, and A. Mallet, Intermittency and alignment in strong RMHD turbulence, Astrophys. J. 807, 39 (2015).

[27] A. Mallet and A. A. Schekochihin, A statistical model of three-dimensional anisotropy and intermittency in strong Alfvénic turbulence, Mon. Not. R. Astron. Soc. 466, 3918 (2017).

[28] O. Alexandrova, V. Carbone, P. Veltri, and L. Sorriso-Valvo, Small-scale energy cascade of the solar wind turbulence, Astrophys. J. 674, 1153 (2008).

[29] F. Sahraoui, M. L. Goldstein, P. Robert, and Yu. V. Khotyaintsev, Evidence of a Cascade and Dissipation of Solar-Wind Turbulence at the Electron Gyroscale, Phys. Rev. Lett. 102, 231102 (2009).

[30] C. H. K. Chen, T. S. Horbury, A. A. Schekochihin, R. T. Wicks, O. Alexandrova, and J. Mitchell, Anisotropy of Solar Wind Turbulence between Ion and Electron Scales, Phys. Rev. Lett. 104, 255002 (2010).

[31] O. Alexandrova, C. Lacombe, A. Mangeney, R. Grappin, and M. Maksimovic, Solar wind turbulent spectrum at plasma kinetic scales, Astrophys. J. 760, 121 (2012).

[32] C. H. K. Chen, S. Boldyrev, Q. Xia, and J. C. Perez, Nature of Subproton Scale Turbulence in the Solar Wind, Phys. Rev. Lett. 110, 225002 (2013).

[33] A. A. Schekochihin, S. C. Cowley, W. Dorland, G. W. Hammett, G. G. Howes, E. Quataert, and T. Tatsuno, Astrophysical gyrokinetics: Kinetic and fluid turbulent cascades in magnetized weakly collisional plasmas, Astrophys. J. Suppl. Ser. 182, 310 (2009).

[34] S. Boldyrev and J.C. Perez, Spectrum of kinetic-Alfvén turbulence, Astrophys. J. 758, L44 (2012).

[35] L. Franci, S. Landi, L. Matteini, A. Verdini, and P. Hellinger, High-resolution hybrid simulations of kinetic plasma turbulence at proton scales, Astrophys. J. 812, 21 (2015).

[36] L. Franci, S. Landi, L. Matteini, A. Verdini, and P. Hellinger, Plasma beta dependence of the ion-scale spectral break of solar wind turbulence: High-resolution 2D hybrid simulations, Astrophys. J. 833, 91 (2016).

[37] D. Grošelj, A. Mallet, N. F. Loureiro, and F. Jenko, Fully Kinetic Simulation of 3D Kinetic Alfvén Turbulence, Phys. Rev. Lett. 120, 105101 (2018).

[38] C. W. Smith, K. Hamilton, B. J. Vasquez, and R. J. Leamon, Dependence of the dissipation range spectrum of interplanetary magnetic fluctuations on the rate of energy cascade, Astrophys. J. Lett. 645, L85 (2006).

[39] R. Bruno, L. Trenchi, and D. Telloni, Spectral slope variation at proton scales from fast to slow solar wind, Astrophys. J. Lett. 793, L15 (2014).

[40] S. Lion, O. Alexandrova, and A. Zaslavsky, Coherent events and spectral shape at ion kinetic scales in the fast solar wind turbulence, Astrophys. J. 824, 47 (2016). 
[41] S. A. Markovskii, B. J. Vasquez, and C. W. Smith, Statistical analysis of the high-frequency spectral break of the solar wind turbulence at $1 \mathrm{AU}$, Astrophys. J. 675, 1576 (2008).

[42] B. D. G. Chandran, B. Li, B. N. Rogers, E. Quataert, and K. Germaschewski, Perpendicular ion heating by lowfrequency Alfvén-wave turbulence in the solar wind, Astrophys. J. 720, 503 (2010).

[43] A. Mallet, A. A. Schekochihin, and B. D. G. Chandran, Disruption of Alfvénic turbulence by magnetic reconnection in a collisionless plasma, J. Plasma Phys. 83, 905830609 (2017).

[44] N. F. Loureiro and S. Boldyrev, Role of Magnetic Reconnection in Magnetohydrodynamic Turbulence, Phys. Rev. Lett. 118, 245101 (2017).

[45] D. Vech, A. Mallet, K. G. Klein, and J. C. Kasper, Magnetic reconnection may control the ion-scale spectral break of solar wind turbulence, Astrophys. J. Lett. 855, L27 (2018).

[46] Y. Voitenko and J. De Keyser, MHD-kinetic transition in imbalanced Alfvénic turbulence, Astrophys. J. Lett. 832, L20 (2016).

[47] O. Alexandrova. Solar wind vs. magnetosheath turbulence and Alfvén vortices, Nonlinear Processes Geophys. 15, 95 (2008).

[48] D. Perrone, O. Alexandrova, A. Mangeney, M. Maksimovic, C. Lacombe, V. Rakoto, J. C. Kasper, and D. Jovanovic, Compressive coherent structures at ion scales in the slow solar wind, Astrophys. J. 826, 196 (2016).

[49] G. K. Batchelor, The Theory of Homogeneous Turbulence (Cambridge University Press, Cambridge, England, 1953).

[50] A. Mallet, K. G. Klein, B. D. G. Chand ran, D. Grošelj, I. W. Hoppock, T. A. Bowen, C. S. Salem, and S. D. Bale, Interplay between intermittency and dissipation in collisionless plasma turbulence, J. Plasma Phys. 85, 175850302 (2019).

[51] B. J. Vasquez, C. W. Smith, K. Hamilton, B. T. MacBride, and R.J. Leamon, Evaluation of the turbulent energy cascade rates from the upper inertial range in the solar wind at 1 AU, J. Geophys. Res. 112, A07101 (2007).

[52] B. T. MacBride, C. W. Smith, and M. A. Forman, The turbulent cascade at $1 \mathrm{AU}$ : Energy transfer and the thirdorder scaling for MHD, Astrophys. J. 679, 1644 (2008).

[53] J. E. Stawarz, C. W. Smith, B. J. Vasquez, M. A. Forman, and B. T. MacBride, The turbulent cascade and proton heating in the solar wind at $1 \mathrm{AU}$, Astrophys. J. 697, 1119 (2009).

[54] P. Hellinger, P. M. TráVníček, Š. Štverák, L. Matteini, and M. Velli, Proton thermal energetics in the solar wind: Helios reloaded, J. Geophys. Res. 118, 1351 (2013).

[55] B. D. G. Chandran and J. C. Perez, Reflection-driven magnetohydrodynamic turbulence in the solar atmosphere and solar wind, J. Plasma Phys. 85, 905850409 (2019).

[56] R. Bandyopadhyay et al., Enhanced energy transfer rate in solar wind turbulence observed near the Sun from Parker Solar Probe, Astrophys. J. Suppl. Ser. 246, 48 (2020).

[57] G. G. Howes, J. M. Tenbarge, W. Dorland, E. Quataert, A. A. Schekochihin, R. Numata, and T. Tatsuno, Gyrokinetic Simulations of Solar Wind Turbulence from Ion to Electron Scales, Phys. Rev. Lett. 107, 035004 (2011).
[58] O. Alexandrova, J. Saur, C. Lacombe, A. Mangeney, J. Mitchell, S. J. Schwartz, and P. Robert, Universality of Solar-Wind Turbulent Spectrum from MHD to Electron Scales, Phys. Rev. Lett. 103, 165003 (2009).

[59] T. Passot and P. L. Sulem, A model for the non-universal power law of the solar wind sub-ion-scale magnetic spectrum, Astrophys. J. Lett. 812, L37 (2015).

[60] N. J. Fox, M. C. Velli, S. D. Bale, R. Decker, A. Driesman, R. A. Howard, J. C. Kasper, J. Kinnison, M. Kusterer, D. Lario, M. K. Lockwood, D. J. McComas, N. E. Raouafi, and A. Szabo, The solar probe plus mission: Humanity's first visit to our star, Space Sci. Rev. 204, 7 (2016).

[61] S. D. Bale et al., The FIELDS Instrument Suite for solar probe plus. Measuring the coronal plasma and magnetic field, plasma waves and turbulence, and radio signatures of solar transients, Space Sci. Rev. 204, 49 (2016).

[62] J. C. Kasper et al., Solar wind electrons alphas and protons (sweap) investigation: Design of the solar wind and coronal plasma instrument suite for solar probe plus, Space Sci. Rev. 204, 131 (2016).

[63] M. M. Martinović et al., The enhancement of proton stochastic heating in the near-sun solar wind, Astrophys. J. Suppl. Ser. 246, 30 (2020).

[64] M. D. McManus, T. A. Bowen, A. Mallet, C. H. K. Chen, B. D. G. Chandran, S. D. Bale, D. E. Larson, T. Dudok de Wit, J. C. Kasper, M. Stevens, P. Whittlesey, R. Livi, K. E. Korreck, K. Goetz, P. R. Harvey, M. Pulupa, R. J. MacDowall, D. M. Malaspina, A. W. Case, and J.W. Bonnell, Cross helicity reversals in magnetic switchbacks, Astrophys. J. Suppl. Ser. 246, 67 (2020).

[65] T. N. Parashar et al., Measures of scale-dependent Alfvénicity in the first PSP solar encounter, Astrophys. J. Suppl. Ser. 246, 58 (2020).

[66] S. D. Bale, S. T. Badman, J. W. Bonnell, T. A. Bowen, D. Burgess, A. W. Case, C. A. Cattell, B. D. G. Chandran, C. C. Chaston, C. H. K. Chen et al., Highly structured slow solar wind emerging from an equatorial coronal hole, Nature (London) 576, 237 (2019).

[67] D. Duan, T. A. Bowen, C. H. K. Chen, A. Mallet, J. He, S. D. Bale, D. Vech, J. C. Kasper, M. Pulupa, J. W. Bonnell, A. W. Case, T. Dudok de Wit, K. Goetz, P. R. Harvey, K. E. Korreck, D. Larson, R. Livi, R. J. MacDowall, D. M. Malaspina, M. Stevens, and P. Whittlesey, The radial dependence of proton-scale magnetic spectral break in slow solar wind during PSP encounter 2, Astrophys. J. Suppl. Ser. 246, 55 (2020).

[68] D. Vech, J. C. Kasper, K. G. Klein, J. Huang, M. L. Stevens, C. H. K. Chen, A. W. Case, K. Korreck, S. D. Bale, T. A. Bowen, P. L. Whittlesey, R. Livi, D. E. Larson, D. Malaspina, M. Pulupa, J. Bonnell, P. Harvey, K. Goetz, T. Dudok de Wit, and R. MacDowall, Kinetic-scale spectral features of cross helicity and residual energy in the inner heliosphere, Astrophys. J. Suppl. Ser. 246, 52 (2020).

[69] T. A. Bowen, S. D. Bale, J. W. Bonnell, T. Dudok de Wit, K. Goetz, K. Goodrich, J. Gruesbeck, P. R. Harvey, G. Jannet, A. Koval, R. J. MacDowall, D. M. Malaspina, M. Pulupa, C. Revillet, D. Sheppard, and A. Szabo, A merged searchcoil and fluxgate magnetometer data product for Parker Solar Probe Fields, J. Geophys. Res. SPACE 125, e2020JA027813 (2020). 
[70] S. T. Badman et al., Magnetic connectivity of the ecliptic plane within $0.5 \mathrm{au}$ : Potential field source surface modeling of the first Parker Solar Probe encounter, Astrophys. J. Suppl. Ser. 246, 23 (2020).

[71] T. A. Bowen et al., Ion-scale Electromagnetic waves in the inner heliosphere, Astrophys. J. Suppl. Ser. 246, 66 (2020).

[72] A. W. Case et al., The solar probe cup on the Parker Solar Probe, Astrophys. J. Suppl. Ser. 246, 43 (2020).

[73] P. L. Whittlesey, D. E. Larson, J. C. Kasper, J. Halekas, M. Abatcha, R. Abiad, M. Berthomier, A. W. Case, J. Chen, D. W. Curtis, G. Dalton, K. G. Klein, K. E. Korreck, R. Livi, M. Ludlam, M. Marckwordt, A. Rahmati, M. Robinson, A. Slagle, M. L. Stevens, C. Tiu, and J. L. Verniero. The solar probe analyzers-electrons on the Parker Solar Probe, Astrophys. J. Suppl. Ser. 246, 74 (2020).

[74] J. S. Halekas, P. Whittlesey, D. E. Larson, D. McGinnis, M. Maksimovic, M. Berthomier, J. C. Kasper, A. W. Case, K. E. Korreck, M. L. Stevens, K. G. Klein, S. D. Bale, R. J. MacDowall, M. P. Pulupa, D. M. Malaspina, K. Goetz, and P. R. Harvey, Electrons in the young solar wind: First results from the Parker Solar Probe, Astrophys. J. Suppl. Ser. 246, 22 (2020).

[75] A. Hasegawa, Particle acceleration by MHD surface wave and formation of aurora, J. Geophys. Res. 81, 5083 (1976).

[76] R. L. Lysak and W. Lotko, On the kinetic dispersion relation for shear Alfvén waves, J. Geophys. Res. 101, 5085 (1996).

[77] J. V. Hollweg, Kinetic Alfvén wave revisited, J. Geophys. Res. 104, 14811 (1999).

[78] G. G. Howes, S. C. Cowley, W. Dorland, G. W. Hammett, E. Quataert, and A. A. Schekochihin, Astrophysical gyrokinetics: Basic equations and linear theory, Astrophys. J. 651, 590 (2006).

[79] M. L. Goldstein, D. A. Roberts, and C. A. Fitch, Properties of the fluctuating magnetic helicity in the inertial and dissipation ranges of solar wind turbulence, J. Geophys. Res. 99, 11519 (1994).
[80] H. Li, S. P. Gary, and O. Stawicki, On the dissipation of magnetic fluctuations in the solar wind, Geophys. Res. Lett. 28, 1347 (2001).

[81] A. Schreiner and J. Saur, A model for dissipation of solar wind magnetic turbulence by kinetic Alfvén waves at electron scales: Comparison with observations, Astrophys. J. 835, 133 (2017).

[82] R. J. Leamon, C. W. Smith, N. F. Ness, and H. K. Wong, Dissipation range dynamics: Kinetic Alfvén waves and the importance of $\beta_{e}$, J. Geophys. Res. 104, 22331 (1999).

[83] J. L. Verniero et al., Parker Solar Probe observations of proton beams simultaneous with ion-scale waves, Astrophys. J. Suppl. Ser. 248, 5 (2020).

[84] P. A. Isenberg, The kinetic shell model of coronal heating and acceleration by ion cyclotron waves: 3 . The proton halo and dispersive waves, J. Geophys. Res. 109, A03101 (2004).

[85] J. He, L. Wang, C. Tu, E. Marsch, and Q. Zong, Evidence of Landau and cyclotron resonance between protons and kinetic waves in solar wind turbulence, Astrophys. J. Lett. 800, L31 (2015).

[86] H. Politano and A. Pouquet, Von Kármán-Howarth equation for magnetohydrodynamics and its consequences on thirdorder longitudinal structure and correlation functions, Phys. Rev. E 57, R21 (1998).

[87] H. Politano and A. Pouquet, Dynamical length scales for turbulent magnetized flows, Geophys. Res. Lett. 25, 273 (1998).

[88] L. Sorriso-Valvo et al., Turbulence-Driven Ion Beams in the Magnetospheric Kelvin-Helmholtz Instability, Phys. Rev. Lett. 122, 035102 (2019).

[89] G. Miloshevich, T. Passot, and P. L. Sulem, Modeling imbalanced collisionless Alfvén wave turbulence with nonlinear diffusion equations, Astrophys. J. Lett. 888, L7 (2020).

[90] K. T. Osman, W. H. Matthaeus, A. Greco, and S. Servidio, Evidence for inhomogeneous heating in the solar wind, Astrophys. J. Lett. 727, L11 (2011). 